Review

\title{
Multidimensional Roles of Collagen Triple Helix Repeat Containing 1 (CTHRC1) in Malignant Cancers
}

\author{
Neng Jiang ${ }^{*}$, YongMei Cui ${ }^{*}$, JunXiu $\mathrm{Liu}^{2}$, XiaoLin $\mathrm{Zhu}^{3}$, Hui Wu ${ }^{4}$, Zheng Yang ${ }^{1}, \mathrm{ZunFu} \mathrm{Ke}{ }^{1 凶}$ \\ 1. Department of Pathology, the First Affiliated Hospital, Sun Yat-sen University, Guangzhou, Province Guangdong, P.R. China; \\ 2. Department of Gynecology, the First Affiliated Hospital, Sun Yat-sen University, Guangzhou, Province Guangdong, P.R. China; \\ 3. Department of Otolaryngology, the First Affiliated Hospital, Sun Yat-Sen University, Guangzhou, Province Guangdong, P.R. China; \\ 4. Department of Gastrointestinal Surgery, the First Affiliated Hospital, Sun Yat-sen University, Guangzhou, Province Guangdong, P.R. China. \\ *These authors contributed equally to this paper. \\ $\triangle$ Corresponding author: Dr. ZunFu Ke, E-mail: kezunfu@126.com.
}

() Ivyspring International Publisher. Reproduction is permitted for personal, noncommercial use, provided that the article is in whole, unmodified, and properly cited. See http://ivyspring.com/terms for terms and conditions.

Received: 2016.06.20; Accepted: 2016.09.04; Published: 2016.10.25

\begin{abstract}
Tumor is one of the principal diseases that seriously threaten human health. Insight into sensitive cancer markers may open a new avenue for the early diagnosis and treatment of this disease. CTHRCI has been identified as a cancer-related gene. It is a secretory glycoprotein that possesses multidimensional roles associated with wound repair, bone remodeling, hepatocytes fibrosis, adipose tissue formation, and so on. Our previous studies and numerous reports from other researchers have revealed that the ascended expression of $\mathrm{CTHRCl}$ tends to go hand in hand with tumorigenesis, proliferation, invasion and metastasis in various human malignancies through a series of molecular mechanisms and signaling pathways. However, the detailed pathogenic mechanisms of CTHRCl overexpression in human malignant cancers are not yet clear. Here, we shall focus our description on the functions, expression profile in several representative malignant tumors and a number of molecular mechanisms and signaling pathways involved with $\mathrm{CTHRCl}$. This introductory discussion of CTHRCl will serve as a reference for further research in understanding this intriguing cancer-related protein.
\end{abstract}

Key words: CTHRC1, cancers, signaling pathways, markers, treatment

\section{Introduction}

CTHRC1, identified as an oncogenic protein, was first discovered as a differentially expressed gene in animal models when researchers compared damaged blood vessels with normal rat arteries [1]. This protein is a conserved $28-\mathrm{kDa}$ secreted glycoprotein among vertebrates with 12 Gly-X-Y repeats in a short collagen motif. The human CTHRC1 possesses $92 \%$ amino acid sequence similar to the rat protein [2]. CTHRC1 was originally detected to be transiently induced in injured arteries [1]. Enhanced expression of CTHRC1 was proven to reverse collagen synthesis dramatically, improve cell migration in rat fibroblasts, smooth muscle cells, and increase neointimal formation to promote vascular and tissue repair process [1]. Similarly, inhibiting collagen matrix deposition facilitated the underlying therapeutic value in anti-fibrotic therapy of keloids [3]. Some ongoing studies showed that CTHRC1 expression was remarkably related to Hepatitis B virus (HBV) infection and increased expression of CTHRC1 helped HBV replication by several signaling cascades [4, 5]. In addition, some other studies have demonstrated that established liver fibrosis could be attenuated and reversed through promoting the expression of CTHRC1 [4, 6]. Empirical evidence also indicated that up-regulation of CTHRC1 served as a positive regulatory factor for bone formation and bone remodeling [7, 8]. Furthermore, CTHRC1 was detected in calcifying atherosclerotic plaques of human blood vessels [1]. A recent study 
exhilaratingly elaborated the significant impacts of CTHRC1 in the regulation of adipose tissue formation, cellular glycogen levels, body composition, muscle function and physical activity $[9,10]$. Interestingly, CTHRC1 is a secretory protein that has been regarded as a kind of meaningful circulating hormone for cell physiology and metabolism [10]. Aberrant expression of CTHRC1, in particular, is ubiquitously associated with cancer proliferation, invasion, metastasis, clinical stages, tumor size, nodal metastasis, TNM pathologic stage and menopause. Thus, up-regulation of CTHRC1 was a positive indicator of poor prognosis in malignant tumor patients [11-15]. In previous reports, the tumor microenvironment of extracellular matrix (ECM) and epithelial-mesenchymal transition (EMT), which are significantly related to the aggressive feature of cancer cells $[12,16-18]$, has been suggested to be mediated by CTHRC1 [12, 15]. Excavated molecular biology characterizations reveal that the expression level of CTHRC1 significantly correlates with transforming growth factor- $\beta$ (TGF- $\beta 1$ ) and promoter demethylation [13, 19]. Furthermore, N-glycosylation may cooperate with canonical Wnt signaling pathways to intensify the overexpression of CTHRC1 and promote Oral squamous cell carcinoma (OSCC) metastasis [20].

However, the potential mechanisms have not been illustrated perfectly. We have already published two articles in the journal Oncotarget, elucidating the association of aberrant expression of CTHRC1 with increased tumor invasion, metastasis and prognosis $[11,12]$. In this review, we shall summarize almost all of the related functions known to have involved with CTHRC1 to identify the ubiquitous role of CTHRC1 in CTHRC1-associated tumors.

\section{Expression profile in several representative malignant tumors}

A series of clinical studies have convincingly demonstrated that CTHRC1 protein and mRNA expression were ubiquitously detected in many human solid tumors [2], and that CTHRC1 overexpression in tumor cells increased the characteristics of malignant aggressiveness including increased tumor growth, invasion and metastasis. These researches have suggested that CTHRC1 may be developed as a potential crucial biomarker of poor outcome and a potential molecular target for anticancer therapies. Here, we would center our description on several representative malignant tumors and several molecular mechanisms to dilacerate the mask of this polyhedral cancer-related protein and to explore the underlying therapeutic targets in malignant cancer patients.

\section{Non-small cell lung cancer (NSCLC)}

Recent research has explored the aberrant up-regulation of CTHRC1 in NSCLC [11, 21, 22], Ke al el showed that overexpression of CTHRC1 increased NSCLC proliferation, invasion and metastasis in vitro via the Wnt/ $\beta$-catenin signaling pathway [11]. Based on this observation, paraffin-embedded immunohistochemical analysis of CTHRC1 in NSCLC patient tissues $(212 / 292,72.60 \%)$ illustrated that the up-regulated levels of both mRNA and protein were evidently higher than those in the paired adjacent non-cancerous tissues $(6 / 66,9.09 \%) \quad(p<0.001)$. Likewise, western blot, RT-PCR and immunofluorescence analyses results indicated that the expression of CTHRC1 in five NSCLC cell lines (NCI-H226, NCl-H23, NCl-H820, NCl-H446 and A549) was markedly stronger than that of normal human lung epithelial cells (Beas-2Bs) [11]. Two independent analyses using lung cancer specimens revealed that overexpression of CTHRC1 strikingly associated with differentiation degree, clinical stage, $\mathrm{T}$ classification, lymph node metastasis, distant metastasis and cigarette smoke as well as with poor clinical survival time of the patients compared with patient with low CTHRC1 expression [11, 21]. Furthermore, the observed results displayed that there were no significant differences between CTHRC1 and mRNA levels with different genders, ages or pathological types [22]. Particularly, an ongoing study revealed that CTHRC1 interacted with microRNA-30b to decrease the ability of invasion and migration in NSCLC patients [22]. These results suggest that CTHRC1 overexpression is a valuable prognosis biomarker for NSCLC.

\section{Colorectal cancer (CRC)}

Similar to the NSCLC studies, CTHRC1 is a CRC-related protein that is differentially expressed between normal human tissues and tumor patient tissues [13]. In a study, up-regulation of CTHRC1 increased the ability of invasiveness and proliferation in colon cancer cell lines and cancer cells. Comparing CTHRC1 with low and high-expression groups, the average recurrence rates were strongly different between low-expression (22\%) and high-expression groups $(44 \%)(p=0.026)$ [13]. Furthermore, researchers were surprised to discover that enhanced expression of CTHRC1 showed close relevance to peritoneal carcinomatosis (PC) and indicated poor prognosis in CRC patients [23]. Moreover, CTHRC1 mediated matrix metalloproteinase 9 (MMP9) expression, which, during breakage from the border of the primary tumor, resulted in invasiveness and metastasis promotion [24-26], by activating ERK signaling pathway [13]. In addition, Hypomethylation 
of DNA has been revealed to induce tumorigenesis through several mechanisms [27, 28], with increasing evidence suggesting that the frequent aberrant overexpression of CTHRC1 protein in colon cancer may be attributed to the exon 1 region demethylation of CTHRC1 gene [13]. These data clearly elaborated that CTHRC1 overexpression is a promising prognostic biomarker of CRC progression associated with poor survival rate of the patients.

\section{Epithelial ovarian cancer (EOC)}

CTHRC1 is up-regulated in EOC tissues and cell lines compared with borderline malignancy and benign tissue and normal ovarian epithelial cell lines [12]. In order to investigate CTHRC1 expression profile and clinical and pathologic features in EOC, a recent article has suggested that CTHRC1 upregulation plays a crucial role to promote malignant cancer invasiveness and metastasis progression in EOC. Western blots and immunohistochemical results revealed that CTHRC1 expression was up-regulated in all eight pairs of fresh EOC tissues compared to that of their corresponding adjacent ovarian tissues. Additionally, another paraffin-embedded immunohistochemical analysis of CTHRC1 in 88 EOC patients' tissue specimens, compared with that in 17 ovarian borderline tissue specimens and 22 ovarian benign epithelial tissue specimens showed that CTHRC1 staining intensity gradually increased in accordance with malignancy: from benign, borderline, early stage to advanced stage $(p<0.001)$. Similar conclusions at the mRNA levels were observed by real-time PCR. Also, CTHRC1 was significantly stronger in EOC patients with a marked correlation with cancer size, menopause, clinical stage, lymphatic metastasis and poor free and overall survival of EOC patients. Collectively, these data indicated that there was no correlation of CTHRC1 up-regulation with age, tumor grades and tumor subtypes. Moreover, in a series of cell function assays involved in EOC cell lines, Hou et al demonstrated that CTHRC1 overexpression correlated with clinicopathologic characters including cell adhesion, proliferation, invasiveness, metastasis. Furthermore, these findings uncovered CTHRC1 up-expression leading to strong decline in the levels of E-cadherin, decreasing expression of E-cadherin as one of the prototypical biomarkers of EMT, through activating the signal pathway of $\mathrm{Wnt} / \beta$-catenin. Coincident with these observations, this study has found that the activation level of $\mathrm{Wnt} / \beta$-catenin signal and metastatic ability were decreased in EOC patients treated by Dkk1, a specific antagonist of Wnt/ $\beta$-catenin signal [29]. Interestingly, data also elucidated higher CTHRC1 protein levels in postmenopausal women compared with premenopausal women [12].

\section{Pancreatic ductal adenocarcinomas (PDAC)}

Consistent with current study, the levels of CTHRC1 messenger RNA and genome expression information of transcripts were compared between PDAC and normal pancreas, results showed a statistically significant enhancement in PDAC tissue samples: $77 \%$ of pancreatic tumors (23 out of 30 ) suggested strong staining in the cell membrane and cytoplasm. Migration and adhesiveness function experiment of pancreatic cancer cells showed that, up-expression of CTHRC1 in pancreatic cancer cells can enhance the abilities of migration and adhesiveness. Oppositely, down-regulation of CTHRC1 in pancreatic cancer cell lines decreased cell adhesiveness and metastasis. In conclusion, overexpression of CTHRC1 in PDAC plays irreplaceable roles in the progression and invasion of this disease. Orthotopic xenograft tumor mouse models demonstrated the role of CTHRC1 could promote PDAC metastasis from primary location to distant organs, such as liver, spleen, stomach, colon and diaphragm. The incremental levels of CTHRC1 expression in PDAC led to strengthened adhesiveness and motility. In further investigation into the possible molecular mechanism of CTHRC1 involvement in the acceleration of cellular migration and adhesiveness, recent works have elaborated that CTHRC1 is required for regulating human pancreatic cancer cells growth and migration through a series of signaling molecules, such as the Wnt5a signaling cascade and Src-FAK signaling pathway [30].

\section{Other tumors}

CTHRC1 gene was found to be commonly overexpressed in numerous types of human malignant cancers, such as invasive melanoma, thyroid, cervix, gastric cancer (GC), oral squamous cell carcinoma (OSCC), breast carcinomas, hepatocellular carcinoma (HCC), gastrointestinal tract cancer, dermatofibrosarcoma protuberans (DFSP), and so on [2, 4, 13, 19, 31-33]. The differential expression analysis of testicular, prostate and tracheal tumors showed no statistical significance [2]. The observation of the frequent expression in DFSP and negative expression in dermatofibroma (DF) can be used as a label for the differential diagnosis of these two diseases [33]. CTHRC1 acts as a tumor promoter in most of them. Overall, CTHRC1 overexpression is strongly correlated with cancer and is a promising target for cancer therapeutics. 


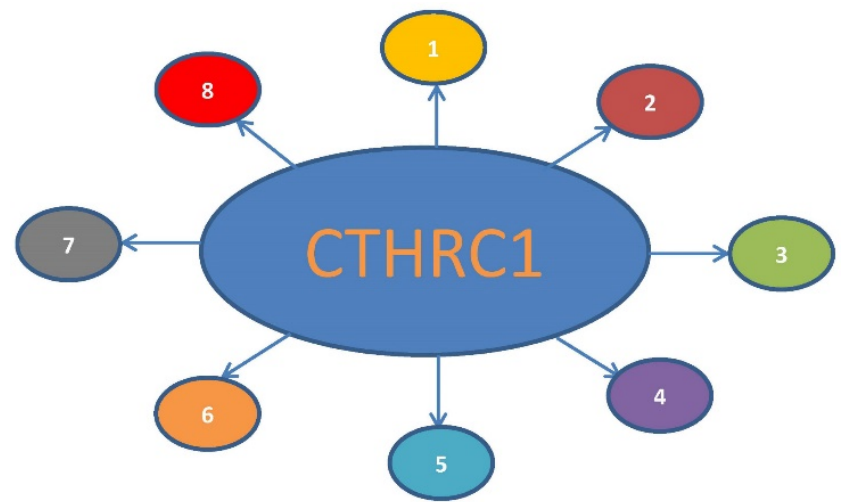

Figure 1. Primary function profile of CTHRC1. 1. Cancer-related protein, a valuable prognostic factor. 2 . Reversing collagen synthesis, increasing the ability of cell migration. 3. Promoting vascular and tissue repair process. 4. Preventing fibrosis of liver and keloid. 5. Closely related to HBV replication. 6. Bone formation and bone remodeling. 7. Relevance with atherosclerosis. 8. Circulating hormone for metabolism and physiology.

\section{The role of CTHRC1 in multi-signaling pathways}

\section{TGF- $\beta$ and BMP4 (bone morphogenetic protein-4) signaling pathway}

It has been reported that CTHRC1 is mainly regulated by TGF- $\beta$ and BMP4 signaling pathways [1], and there is evidence showing that TGF- $\beta$ signaling is involved in the occurrence and development of tumor [34-36]. Meanwhile, the inordinate TGF- $\beta$ signaling activation has been found in many solid cancers. CTHRC1 mRNA and protein levels are enhanced in fibroblasts and chondrocytic cells in response to TGF- $\beta$ family members [37], and there are many lines of evidence indicating that TGF- $\beta$ regulates the expression level of CTHRC1 in a timeand concentration-dependent manner $[3,19]$. As we know, TGF- $\beta$ signaling pathway mediates multiple regulatory factors for vessel repair such as collagen type I and III. Nevertheless, it has been suggested that CTHRC1 could up-regulate in fibroblasts and smooth muscle cells of blood vessels, which indicates that CTHRC1 may has the ability to inhibit the deposition of collagen type I and III, which leads to the acceleration of cell migration in case of vascular remodeling $[1,38,39]$. Interestingly, promoter region of CTHRC1 has a binding site of Smads which is a downstream receptor of TGF- $\beta /$ BMP4 pathways [19]. Therefore, it is possible that the induction of CTHRC1 is partially due to the activation of TGF- $\beta$ and BMP signaling pathways. BMP4 signaling pathway is similar to TGF- $\beta$, while the downstream cascade of BMP4 involves smad1/5/8 [40]. In a word, TGF- $\beta$ and BMP4 increase CTHRC1 transcription by regulating downstream transcription effectors mainly activated pho-Smads, which can bind to the promoter region of CTHRC1. While highly expressed CTHRC1 inhibits the activation of Smad in 293T cell line, it did not work in some other cell types [41]. Consistent with this, Pyagay reported that the upregulation of CTHRC1 inhibited collagen deposition, mainly including collagen type I and III, by impairing the phosphorylation activation of Smad2/3 which were the important components of TGF- $\beta$ signaling $[1,42]$. Beside this, understanding the complicated mechanisms between CTHRC1, composition of ECM and TGF- $\beta$ signaling pathway requires further investigation.

\section{Canonical Wnt/ $\beta$-catenin signaling pathway and noncanonical Wnt/PCP pathway}

A number of studies have found that Wnt/ $\beta$-catenin signaling pathway has complicated relationship with many other cellular signaling pathways, which indicates that Wnt/ $\beta$-catenin signaling can be regulated by a mass of cellular molecules. The biological function of $\mathrm{Wnt} / \beta$-catenin signaling pathway refers to cellular metabolism and the early pathogenesis and development of many human cancers [43-45]. In the presence of ligand Wnt3a/1/8, Wnt/ $\beta$-catenin pathway will be activated, including Wnt-FZDs-Lrp5/6 complex which transduces signals to the cellular downstream and leads to accumulation of $\beta$-catenin in cytoplasm. The upregulated $\beta$-catenin translocates to nucleus and regulates a wide range of genes by binding to a series transcription factors such as TCF/LEF [20]. There is scientific evidence suggesting that the biological effect of CTHRC1 on metastasis and proliferation of tumor cells is cross-linked with the canonical Wnt/ $\beta$-catenin pathway. Several studies have investigated the interaction between CTHRC1 and Wnt/ $\beta$-catenin pathway. In one article, luciferase assays demonstrated that expression of CTHRC1 was positively correlated with the transcriptional activity of $\beta$-catenin/TCF, and CTHRC1 had a positive relationship with cellular $\beta$-catenin [20]. According to the research of Oral squamous cell carcinoma (OSCC), activated $\mathrm{Wnt} / \beta$-catenin signaling upregulates the transcription of CTHRC1 by regulating the activity of $\beta$-catenin/TCF in the region of CTHRC1 promoter [11]. At the same time, several studies have revealed that N-glycosylation protein DPAGT1 is connected with Wnt/ $\beta$-catenin signaling [46]. Studies also demonstrated that CTHRC1 expression can be up-regulated by DPAGT1/Wnt/ $\beta$-catenin positive feedback loop. In conclusion, activated Wnt $/ \beta$-catenin signaling pathway upregulates CTHRC1 expression and the DPAGT1/Wnt/ $\beta$ catenin positive feedback loop enhances the function of regulating CTHRC1. While, it is not clear how CTHRC1 regulates $W n t / \beta$-catenin signaling pathway. 
Because of the diversity of Wnt ligands and receptors, Wnt-FZD-coreceptor compound can activate alternate signaling pathway, which transduces cell signals in a different manner compared with classical Wnt/ $\beta$-catenin signaling pathway. Here we introduce Wnt/PCP signaling pathway, one important noncanonical Wnt pathway. Wnt/PCP signaling pathway regulates planar cell polarity and related developmental events by activating downstream signaling molecules such as RhoA, Rac1, JNK and NLK [23, 47]. It has been proved that the activated RhoA and Rac1 cascades promote EMT by changing cell cytoskeleton [48]. Yamamoto et al demonstrated in 2008 that cell-surface-anchored CTHRC1 was associated with Wnt/PCP signaling pathway through promoting and stabilizing the Wnt/Fzd/CTHRC1/Ror2 compound as a cofactor protein. In 293T cells, CTHRC1 stabilizes the interaction of Wnt5A-FZD-Ror2, and activates the Wnt/PCP pathway but not the $W n t / \beta$-catenin signaling pathway [49]. Subsequently, many experimental results are consistent with this opinion. One more thing worth mentioning is that the activated Wnt/PCP pathway increases CTHRC1 expression in turn.

The above evidence indicates the relationship among CTHRC1, Wnt/ $\beta$-catenin signaling pathway and Wnt/PCP signaling pathway is cross-linked.

\section{Integrin $\beta /$ src-Fak and ERK signaling pathways}

In hepatocellular carcinoma (HCC), knocking down of CTHRC1 can suppress cell migration and invasion force in HCC cells [50]. To elucidate the mechanisms, the team performed a qRT-PCR array and the experiment result demonstrated that integrin $\beta$ underwent great changes after the knock down of CTHRC1. It has been reported that integrin $\beta 2 / \beta 3$ are related with tumor metastasis [51-57]. Integrins, composed by $\alpha$ - and $\beta$-subunits, are one type of transmembrane receptors. By binding to components in the ECM, integrins provide platform for a series of molecules, which can mediate multiple biological events including cell differentiation, adhesion, migration and cell survival [58-60]. It is worth noting that integrins can prevent anoikis, a kind of apoptosis $[61,62]$. The downstream molecules of ligand-integrin $\beta$ mainly contain the FAK kinase and Src cascades, which result in the activation of AKT2/ERK protein kinase. As mentioned above, the binding of CTHRC1 onto ligand Wnt5a stabilizes Wnt5a-FZDCTHRC1-Ror2 compound, which attempts to activate paxillin and a series of kinase including MAPK, Erk and MEK $[63,64]$. It has been proved that CTHRC1 could activate Src and Erk and the phosphorylated Src and Erk signaling cascades, which can promote tumor progression [4]. There has been growing evidence showing that the activated ERK interacts with MMP9, which can down-regulate collagen deposition in the ECM and promote tumor metastasis, and finally increase pro-EMT transcription factor sox 2 and snail [65]. On the other hand, the phosphorylated AKT2/ERK could also promote the expression of HIF-1a which leads to high expression of VEGF, a key growth factor of tumor genesis and progression. However, how CTHRC1 regulates integrin $\beta$ remains to be further elucidated.

\section{P53 signaling pathway}

Tumor suppressor P53 is a famous molecule which mediates the balance between proliferation and apoptosis by activating PTEN and p21. The uncontrolled proliferation of cancer cells is an important characteristics of tumor. There is evidence showing that CTHRC1 overexpression promotes cell proliferation $[12,66]$. The investigation between P53 signaling pathway and CTHRC1 needs to be mentioned. HBV-activated CTHRC1 in HCC inhibits the expression of p53, activates AKT by inhibiting the activation of Forkhead box (FOXO) factors and finally downregulates the transactivation of p21 [4]. However, the effect of HBV-activated CTHRC1 on p53 expression is not yet fully understood and further investigations will be required to determine if it's cell-type-specific.

\section{MicroRNAs, LncRNA and CTHRCI}

MicroRNAs can regulate several pathological conditions including cancer $[67,68]$. Naturally, MMP9 and CTHRC1 are related with tumor metastasis according to previous and ongoing research of different types of solid cancers [13, 69]. According to recent findings, miR520d-5p can directly target CTHRC1. Subsequently, the level of phosphorylated ERK1/2 is reduced subsequently. Otherwise, transcription factor SP1 can transactivate miR520d-5p by binding to its upstream promoter region. In conclusion, SP1-associated microRNA-520d-5p can restrain tumor progression by targeting CTHRC1 in colorectal cancer patients. Bioinformatics analysis elucidated that miR-30b might bind to the 3'UTR region of CTHRC1 and the following result of dual-luciferase reporter assay strongly suggested that the expression of CTHRC1 was down-regulated by miR-30b through binding to 3'UTR [70]. Recently, another paper reported that miR98 could also inhibit the metastasis and invasion of HCC through targeting CTHRC1 directly [71]. Otherwise, MALAT-1, a long non-coding RNA, is also supposed to be related to metastasis and there are results suggesting that MALAT-1 gene silencing reduces cell motility 
through concomitant down-regulation of CTHRC1, HMMR, CCT4 and ROD1 [72].

\section{Discussions}

To date, there has been a decade of research on CTHRC1 since its original identification [1]. CTHRC1 was known as a positive regulator involved in a variety of pathological and normal physiological processes such as anti-fibrosis, metabolism and physiology, tissue repair remodeling, osteoblastic bone formation, and cancer [1-3, 6-8, 10]. Notably, aberrant expression of CTHRC1 was found in different types of cancers, and its overexpression results in increased cancer cell proliferation, invasion and metastasis. CTHRC1 has been recognized as a promising diagnostic biomarker and precision medicine target for human malignant tumors and may play significant roles in different human malignant tumors. The TGF- $\beta$ and BMP, Integrin $\beta /$ src-Fak and ERK, P53 and Wnt signaling pathways have been elucidated to be closely related to the function of CTHRC1. Moreover, promoter demethylation and $\mathrm{N}$-glycosylation also contribute to the protein expression of CTHRC1 in tumors $[19,20]$. Nevertheless, it requires further studies to better understand whether the cellular localization and proteolytic processing, such as ubiquitination and phosphorylation, and nonresolving inflammation sustained activations are involved in CTHRC1 to promote tumor progression. Based on previous observation, the intersection between CTHRC1 and autoimmune is a new research direction in malignant cancers. However, further investigation is needed to clarify the polymorphism and detailed mechanisms of CTHRC1 in cancers. It is critical to elucidate how the complex molecular mechanisms of CTHRC1 impact cancer development and progression. There is a long path in front of us to put the CTHRC1 protein into clinical application. Additionally, increased evidence suggests that miR-520d-5p, miR-30b, Let-7b and miR-98 suppress tumor progression, invasion and migration in gastrointestinal tumors through combining CTHRC1 receptor [71, 73]. MiR-9 can attenuate Schwann cell migration through down-regulating CTHRC1 [74]. The initial biochemical and functional characterization, how overproduced CTHRC1 decreases extracellular matrix synthesis and impacts the extracellular tumor microenvironment need further exploration at systems biological level. In summary, these related works mentioned above have made CTHRC1 a hot biomarker with much higher sensitivity, specificity, and accuracy for targeting various pathogenic diseases, including cancer.

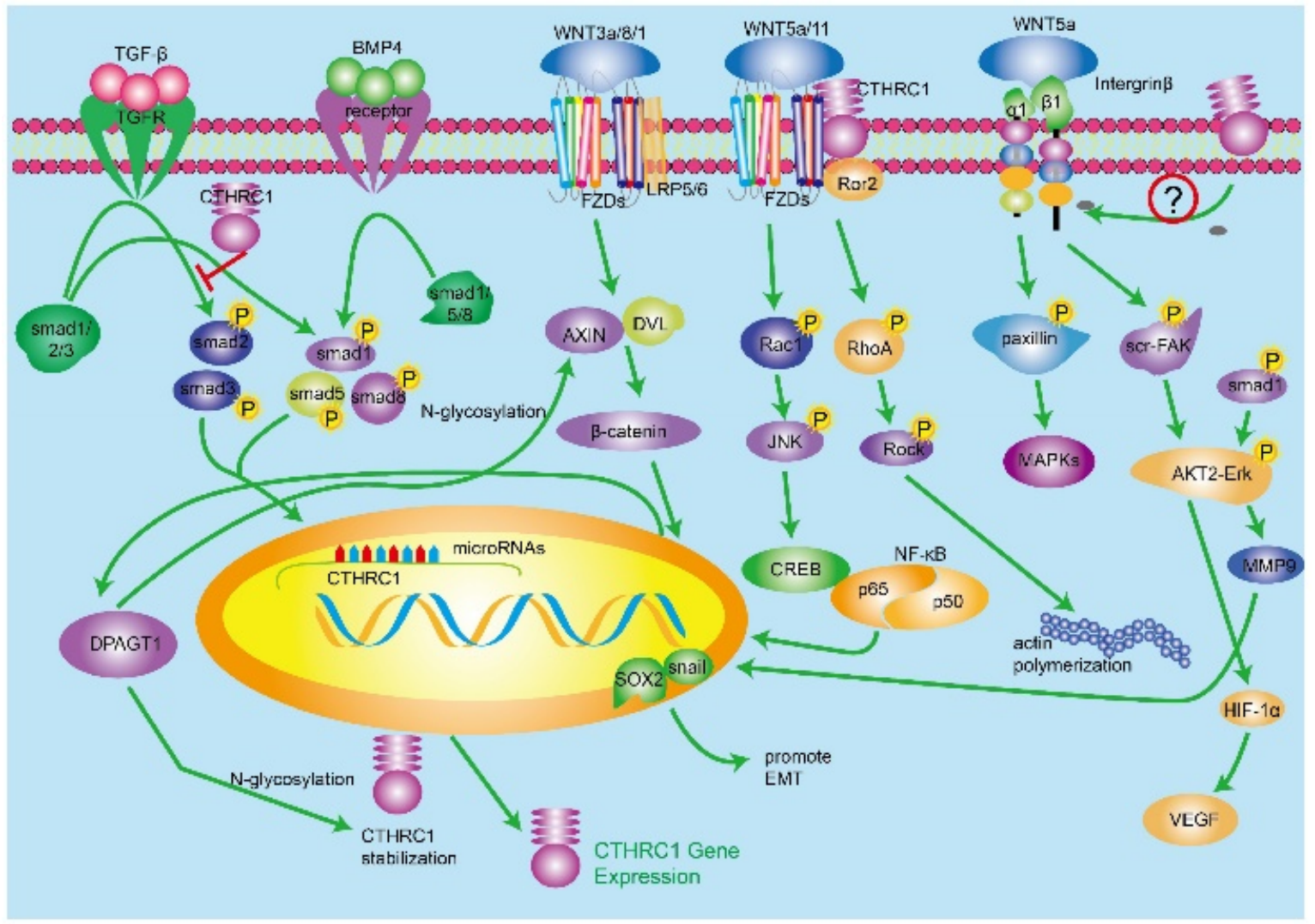

Figure 2. The role of CTHRC1 in multi-signaling pathways. 1. TGF- $\beta$ and BMP4 regulate the expression of CTHRC1. CTHRCl can inhibit the activation of Smad in $293 \mathrm{~T}$ cell line. 2. Canonical Wnt/ $\beta$-catenin signaling pathway and noncanonical Wnt/PCP pathways regulate the expression of CTHRCl by mutiple transcription factors. DPAGT1/canonical Wnt positive feedback loop enhances the function of regulating CTHRC1. 3. The inner interaction between CTHRCl and Integrin $\beta /$ src-Fak/ERK/P53 signaling pathways is not clear so far. 


\section{Competing Interests}

The authors have declared that no competing interest exists.

\section{References}

1. Pyagay P, Heroult M, Wang Q, Lehnert W, Belden J, Liaw L, et al. Collagen triple helix repeat containing 1 , a novel secreted protein in injured and diseased arteries, inhibits collagen expression and promotes cell migration. Circulation research. 2005; 96: 261-8

2. Tang L, Dai DL, Su M, Martinka M, Li G, Zhou Y. Aberrant expression of collagen triple helix repeat containing 1 in human solid cancers. Clinical cancer research : an official journal of the American Association for Cancer Research. 2006; 12: 3716-22.

3. Li J, Cao J, Li M, Yu Y, Yang Y, Xiao X, et al. Collagen triple helix repeat containing-1 inhibits transforming growth factor-b1-induced collagen type I expression in keloid. The British journal of dermatology. 2011; 164: 1030-6.

4. Zhang R, Cao Y, Bai L, Zhu C, Li R, He H, et al. The collagen triple helix repeat containing 1 facilitates hepatitis B virus-associated hepatocellular carcinoma progression by regulating multiple cellular factors and signal cascades. Molecular carcinogenesis. 2015; 54: 1554-66.

5. Bai L, Zhang W, Tan L, Yang H, Ge M, Zhu C, et al. Hepatitis B virus hijacks CTHRC1 to evade host immunity and maintain replication. Journal of molecular cell biology. 2015; 7: 543-56

6. Bian Z, Miao Q, Zhong W, Zhang H, Wang Q, Peng $\mathrm{Y}$, et al. Treatment of cholestatic fibrosis by altering gene expression of Cthrc1: Implications for autoimmune and non-autoimmune liver disease. Journal of autoimmunity. 2015; 63: 76-87.

7. Kimura H, Kwan KM, Zhang Z, Deng JM, Darnay BG, Behringer RR, et al. Cthrc1 is a positive regulator of osteoblastic bone formation. PloS one. 2008; 3 : e3174.

8. Takeshita S, Fumoto T, Matsuoka K, Park KA, Aburatani H, Kato S, et al. Osteoclast-secreted CTHRC1 in the coupling of bone resorption to formation. The Journal of clinical investigation. 2013; 123: 3914-24.

9. Stohn JP, Wang Q, Siviski ME, Kennedy K, Jin YR, Kacer D, et al. Cthrc1 controls adipose tissue formation, body composition, and physical activity. Obesity (Silver Spring, Md). 2015; 23: 1633-42.

10. Stohn JP, Perreault NG, Wang Q, Liaw L, Lindner V. Cthrc1, a novel circulating hormone regulating metabolism. PloS one. 2012; 7: e47142.

11. Ke Z, He W, Lai $Y$, Guo X, Chen S, Li S, et al. Overexpression of collagen triple helix repeat containing 1 (CTHRC1) is associated with tumour aggressiveness and poor prognosis in human non-small cell lung cancer. Oncotarget. 2014; 5: 9410-24.

12. Hou M, Cheng Z, Shen H, He S, Li Y, Pan Y, et al. High expression of CTHRC1 promotes EMT of epithelial ovarian cancer (EOC) and is associated with poor prognosis. Oncotarget. 2015; 6: 35813-29.

13. Kim HC, Kim YS, Oh HW, Kim K, Oh SS, Kim JT, et al. Collagen triple helix repeat containing 1 (CTHRC1) acts via ERK-dependent induction of MMP9 to promote invasion of colorectal cancer cells. Oncotarget. 2014; 5: 519-29.

14. Chen YL, Wang TH, Hsu HC, Yuan RH, Jeng YM. Overexpression of CTHRC1 in hepatocellular carcinoma promotes tumor invasion and predicts poor prognosis. PloS one. 2013; 8: e70324

15. Kim JH, Baek TH, Yim HS, Kim KH, Jeong SH, Kang HB, et al. Collagen triple helix repeat containing-1 (CTHRC1) expression in invasive ductal carcinoma of the breast: the impact on prognosis and correlation to clinicopathologic features. Pathology oncology research : POR. 2013; 19: 731-7.

16. Ma MZ, Zhuang C, Yang XM, Zhang ZZ, Ma H, Zhang WM, et al. CTHRC1 acts as a prognostic factor and promotes invasiveness of gastrointestinal stromal tumors by activating Wnt/PCP-Rho signaling. Neoplasia (New York, NY). 2014; 16: 265-78, 78 e1-13.

17. Gavert N, Ben-Ze'ev A. Epithelial-mesenchymal transition and the invasive potential of tumors. Trends in molecular medicine. 2008; 14: 199-209.

18. Pacheco-Pinedo EC, Durham AC, Stewart KM, Goss AM, Lu MM, Demayo FJ, et al. Wnt/beta-catenin signaling accelerates mouse lung tumorigenesis by imposing an embryonic distal progenitor phenotype on lung epithelium. The Journal of clinical investigation. 2011; 121: 1935-45.

19. Wang P, Wang YC, Chen XY, Shen ZY, Cao H, Zhang YJ, et al. CTHRC1 is upregulated by promoter demethylation and transforming growth factor-beta1 and may be associated with metastasis in human gastric cancer. Cancer science. 2012; 103: 1327-33.

20. Liu G, Sengupta PK, Jamal B, Yang HY, Bouchie MP, Lindner V, et al. $\mathrm{N}$-glycosylation induces the CTHRC1 protein and drives oral cancer cell migration. The Journal of biological chemistry. 2013; 288: 20217-27.

21. Liu X, Liu B, Cui $Y$, Wang F, Sun H, Lv F. Collagen triple helix repeat containing 1 (Cthrc1) is an independently prognostic biomarker of non-small cell lung cancers with cigarette smoke. Tumour biology : the journal of the International Society for Oncodevelopmental Biology and Medicine. 2014; 35: 11677-83.

22. Chen S, Li P, Yang R, Cheng R, Zhang F, Wang Y, et al. microRNA-30b inhibits cell invasion and migration through targeting collagen triple helix repeat containing 1 in non-small cell lung cancer. Cancer cell international. 2015; 15: 85.
23. Tan F, Liu F, Liu H, Hu Y, Liu D, Li G. CTHRC1 is associated with peritoneal carcinomatosis in colorectal cancer: a new predictor for prognosis. Medical oncology (Northwood, London, England). 2013; 30: 473.

24. Gialeli C, Theocharis AD, Karamanos NK. Roles of matrix metalloproteinases in cancer progression and their pharmacological targeting. The FEBS journal. 2011; 278: 16-27.

25. Roy R, Yang J, Moses MA. Matrix metalloproteinases as novel biomarkers and potential therapeutic targets in human cancer. Journal of clinical oncology : official journal of the American Society of Clinical Oncology. 2009; 27: 5287-97.

26. Burg-Roderfeld M, Roderfeld M, Wagner S, Henkel C, Grotzinger J, Roeb E. MMP-9-hemopexin domain hampers adhesion and migration of colorectal cancer cells. International journal of oncology. 2007; 30: 985-92.

27. Robertson KD. DNA methylation, methyltransferases, and cancer. Oncogene. 2001; 20: 3139-55.

28. Costello JF, Plass C. Methylation matters. Journal of medical genetics. 2001; 38: 285-303.

29. Zhang H, Yu C, Dai J, Keller JM, Hua A, Sottnik JL, et al. Parathyroid hormone-related protein inhibits DKK1 expression through c-Jun-mediated inhibition of beta-catenin activation of the DKK1 promoter in prostate cancer. Oncogene. 2014; 33: 2464-77.

30. Park EH, Kim S, Jo JY, Kim SJ, Hwang Y, Kim JM, et al. Collagen triple helix repeat containing-1 promotes pancreatic cancer progression by regulating migration and adhesion of tumor cells. Carcinogenesis. 2013; 34: 694-702.

31. Eriksson J, Le Joncour V, Nummela P, Jahkola T, Virolainen S, Laakkonen P, et al. Gene expression analyses of primary melanomas reveal CTHRC1 as an important player in melanoma progression. Oncotarget. 2016.

32. Lee CE, Vincent-Chong VK, Ramanathan A, Kallarakkal TG, Karen-Ng LP, Ghani WM, et al. Collagen Triple Helix Repeat Containing-1 (CTHRC1) Expression in Oral Squamous Cell Carcinoma (OSCC): Prognostic Value and Clinico-Pathological Implications. International journal of medical sciences. 2015; 12: 937-45.

33. Wang L, Xiang YN, Zhang YH, Tu YT, Chen HX. Collagen triple helix repeat containing-1 in the differential diagnosis of dermatofibrosarcoma protuberans and dermatofibroma. The British journal of dermatology. 2011; 164: 135-40.

34. Hendrix MJ, Seftor EA, Hess AR, Seftor RE. Vasculogenic mimicry and tumour-cell plasticity: lessons from melanoma. Nature reviews Cancer. 2003; 3: 411-21.

35. Teti A, De Giorgi A, Spinella MT, Migliaccio S, Canipari R, Onetti Muda A, et al. Transforming growth factor-beta enhances adhesion of melanoma cells to the endothelium in vitro. International journal of cancer. 1997; 72: 1013-20.

36. Reed JA, McNutt NS, Prieto VG, Albino AP. Expression of transforming growth factor-beta 2 in malignant melanoma correlates with the depth of tumor invasion. Implications for tumor progression. The American journal of pathology. 1994; 145: 97-104.

37. Durmus T, LeClair RJ, Park KS, Terzic A, Yoon JK, Lindner V. Expression analysis of the novel gene collagen triple helix repeat containing-1 (Cthrc1). Gene expression patterns : GEP. 2006; 6: 935-40.

38. Dvorak HF. Tumors: wounds that do not heal. Similarities between tumor stroma generation and wound healing. The New England journal of medicine. 1986; 315: 1650-9.

39. Beachy PA, Karhadkar SS, Berman DM. Tissue repair and stem cell renewal in carcinogenesis. Nature. 2004; 432: 324-31.

40. Kharaishvili G, Cizkova M, Bouchalova K, Mgebrishvili G, Kolar Z, Bouchal J. Collagen triple helix repeat containing 1 protein, periostin and versican in primary and metastatic breast cancer: an immunohistochemical study. Journal of clinical pathology. 2011; 64: 977-82.

41. LeClair R, Lindner V. The role of collagen triple helix repeat containing 1 in injured arteries, collagen expression, and transforming growth factor beta signaling. Trends in cardiovascular medicine. 2007; 17: 202-5.

42. LeClair RJ, Durmus T, Wang Q, Pyagay P, Terzic A, Lindner V. Cthrc1 is a novel inhibitor of transforming growth factor-beta signaling and neointimal lesion formation. Circulation research. 2007; 100: 826-33.

43. Gordon MD, Nusse R. Wnt signaling: multiple pathways, multiple receptors, and multiple transcription factors. The Journal of biological chemistry. 2006; 281: 22429-33

44. van Amerongen R, Nusse R. Towards an integrated view of Wnt signaling in development. Development (Cambridge, England). 2009; 136: 3205-14.

45. Sethi JK, Vidal-Puig A. Wnt signalling and the control of cellular metabolism. The Biochemical journal. 2010; 427: 1-17.

46. Sengupta PK, Bouchie MP, Kukuruzinska MA. N-glycosylation gene DPAGT1 is a target of the Wnt/beta-catenin signaling pathway. The Journal of biological chemistry. 2010; 285 : 31164-73

47. Kelley MW. Leading Wnt down a PCP path: Cthrc1 acts as a coreceptor in the Wnt-PCP pathway. Developmental cell. 2008; 15: 7-8.

48. Chen X, Cheng H, Pan T, Liu Y, Su Y, Ren C, et al. mTOR regulate EMT through RhoA and Rac1 pathway in prostate cancer. Molecular carcinogenesis. 2015; 54: 1086-95.

49. Yamamoto S, Nishimura O, Misaki K, Nishita M, Minami Y, Yonemura S, et al. Cthrc1 selectively activates the planar cell polarity pathway of Wnt signaling by stabilizing the Wnt-receptor complex. Developmental cell. 2008; 15: 23-36.

50. Tameda M, Sugimoto K, Shiraki K, Yamamoto N, Okamoto R, Usui M, et al. Collagen triple helix repeat containing 1 is overexpressed in hepatocellular carcinoma and promotes cell proliferation and motility. International journal of oncology. 2014; 45: 541-8. 
51. Takeichi T, Mocevicius P, Deduchovas O, Salnikova O, Castro-Santa E, Buchler MW, et al. alphaL beta2 integrin is indispensable for CD8+ T-cell recruitment in experimental pancreatic and hepatocellular cancer. International journal of cancer. 2012; 130: 2067-76.

52. Oberyszyn TM, Conti CJ, Ross MS, Oberyszyn AS, Tober KL, Rackoff AI, et al. Beta2 integrin/ICAM-1 adhesion molecule interactions in cutaneous inflammation and tumor promotion. Carcinogenesis. 1998; 19: 445-55.

53. Wu Y, Zuo J, Ji G, Saiyin H, Liu X, Yin F, et al. Proapoptotic function of integrin beta(3) in human hepatocellular carcinoma cells. Clinical cancer research : an official journal of the American Association for Cancer Research. 2009; 15: 60-9.

54. Jeanes AI, Wang P, Moreno-Layseca P, Paul N, Cheung J, Tsang R, et al. Specific beta-containing integrins exert differential control on proliferation and two-dimensional collective cell migration in mammary epithelial cells. The Journal of biological chemistry. 2012; 287: 24103-12.

55. Jung CW, Song TJ, Lee KO, Choi SB, Kim WB, Suh SO, et al. Characterization of hepatocellular carcinoma cell lines based on cell adhesion molecules. International journal of molecular medicine. 2012; 29: 1158-64.

56. Li N, Zhang JP, Guo S, Min J, Liu LL, Su HC, et al. Down-regulation of beta3-integrin inhibits bone metastasis of small cell lung cancer. Molecular biology reports. 2012; 39: 3029-35.

57. Papas MG, Karatzas PS, Papanikolaou IS, Karamitopoulou E, Delicha EM, Adler A, et al. LFA-1 expression in a series of colorectal adenocarcinomas. Journal of gastrointestinal cancer. 2012; 43: 462-6.

58. Oktay M, Wary KK, Dans M, Birge RB, Giancotti FG. Integrin-mediated activation of focal adhesion kinase is required for signaling to Jun $\mathrm{NH} 2$-terminal kinase and progression through the G1 phase of the cell cycle. The Journal of cell biology. 1999; 145: 1461-9.

59. Clark EA, Brugge JS. Integrins and signal transduction pathways: the road taken. Science (New York, NY). 1995; 268: 233-9.

60. Giancotti FG, Ruoslahti E. Integrin signaling. Science (New York, NY). 1999; 285: 1028-32.

61. Maubant S, Saint-Dizier D, Boutillon M, Perron-Sierra F, Casara PJ, Hickman JA, et al. Blockade of alpha $\mathrm{v}$ beta3 and alpha $\mathrm{v}$ beta5 integrins by RGD mimetics induces anoikis and not integrin-mediated death in human endothelial cells. Blood. 2006; 108: 3035-44.

62. Benoit YD, Larrivee JF, Groulx JF, Stankova J, Vachon PH, Beaulieu JF. Integrin alpha8beta1 confers anoikis susceptibility to human intestinal epithelial crypt cells. Biochemical and biophysical research communications. 2010; 399: 434-9.

63. Ishibe S, Joly D, Zhu X, Cantley LG. Phosphorylation-dependent paxillin-ERK association mediates hepatocyte growth factor-stimulated epithelial morphogenesis. Molecular cell. 2003; 12: 1275-85.

64. Ishibe S, Joly D, Liu ZX, Cantley LG. Paxillin serves as an ERK-regulated scaffold for coordinating FAK and Rac activation in epithelial morphogenesis. Molecular cell. 2004; 16: 257-67.

65. Lin CY, Tsai PH, Kandaswami CC, Lee PP, Huang CJ, Hwang JJ, et al. Matrix metalloproteinase-9 cooperates with transcription factor Snail to induce epithelial-mesenchymal transition. Cancer science. 2011; 102: 815-27.

66. Yang XM, You HY, Li Q, Ma H, Wang YH, Zhang YL, et al. CTHRC1 promotes human colorectal cancer cell proliferation and invasiveness by activating Wnt/PCP signaling. International journal of clinical and experimental pathology. 2015; 8: 12793-801.

67. Gomes BC, Rueff J, Rodrigues AS. MicroRNAs and Cancer Drug Resistance. Methods in molecular biology (Clifton, NJ). 2016; 1395: 137-62.

68. Humphries B, Wang Z, Yang C. The role of microRNAs in metal carcinogen-induced cell malignant transformation and tumorigenesis. Food and chemical toxicology : an international journal published for the British Industrial Biological Research Association. 2016.

69. Moirangthem A, Bondhopadhyay B, Mukherjee M, Bandyopadhyay A, Mukherjee N, Konar K, et al. Simultaneous knockdown of uPA and MMP9 can reduce breast cancer progression by increasing cell-cell adhesion and modulating EMT genes. Scientific reports. 2016; 6: 21903.

70. Yan L, Yu J, Tan F, Ye GT, Shen ZY, Liu H, et al. SP1-mediated microRNA-520d-5p suppresses tumor growth and metastasis in colorectal cancer by targeting CTHRC1. American journal of cancer research. 2015; 5: 1447-59.

71. Wang CY, Zhang JJ, Hua L, Yao KH, Chen JT, Ren XQ. MicroRNA-98 suppresses cell proliferation, migration and invasion by targeting collagen triple helix repeat containing 1 in hepatocellular carcinoma. Molecular medicine reports. 2016.

72. Tano K, Mizuno R, Okada T, Rakwal R, Shibato J, Masuo Y, et al. MALAT-1 enhances cell motility of lung adenocarcinoma cells by influencing the expression of motility-related genes. FEBS letters. 2010; 584: 4575-80.

73. Yu J, Feng J, Zhi X, Tang J, Li Z, Xu Y, et al. Let-7b inhibits cell proliferation, migration, and invasion through targeting Cthrc1 in gastric cancer. Tumour biology : the journal of the International Society for Oncodevelopmental Biology and Medicine. 2015; 36: 3221-9.

74. Zhou S, Gao R, Hu W, Qian T, Wang N, Ding G, et al. MiR-9 inhibits Schwann cell migration by targeting Cthrc1 following sciatic nerve injury. Journal of cell science. 2014; 127: 967-76. 